\title{
Diagnostic Accuracy of Bone Turnover Markers and Bone Histology in Patients With CKD Treated by Dialysis
}

\author{
Stuart M. Sprague, DO, ${ }^{1}$ Ezequiel Bellorin-Font, $M D,{ }^{2}$ Vanda Jorgetti, $M D, P h D,{ }^{3}$ \\ Aluizio B. Carvalho, MD, PhD, ${ }^{4}$ Hartmut H. Malluche, $M D,{ }^{5}$ Aníbal Ferreira, MD, $P h D,{ }^{6}$ \\ Patrick C. D'Haese, PhD, Tilman B. Drüeke, MD, ${ }^{8}$ Hongyan Du, MB, MS, ${ }^{1}$ \\ Thomas Manley, RN, CRNA, ${ }^{9}$ Eudocia Rojas, MD, ${ }^{2}$ and Sharon M. Moe, MD ${ }^{10}$
}

\footnotetext{
Background: The management of chronic kidney disease-mineral and bone disorder requires the assessment of bone turnover, which most often is based on parathyroid hormone (PTH) concentration, the utility of which remains controversial.

Study Design: Cross-sectional retrospective diagnostic test study.

Setting \& Participants: 492 dialysis patients from Brazil, Portugal, Turkey, and Venezuela with prior bone biopsy and stored $\left(-20^{\circ} \mathrm{C}\right)$ serum.

Index Tests: Samples were analyzed for PTH (intact [iPTH] and whole PTH), bone-specific alkaline phosphatase (bALP), and amino-terminal propeptide of type 1 procollagen (P1NP).

Reference Test: Bone histomorphometric assessment of turnover (bone formation rate/bone surface [BFR/ BS]) and receiver operating characteristic curves for discriminating diagnostic ability.

Results: The biomarkers iPTH and bALP or combinations thereof allowed discrimination of low from nonlow and high from nonhigh BFR/BS, with an area under the receiver operating characteristic curve $>0.70$ but $<0.80$. Using iPTH level, the best cutoff to discriminate low from nonlow BFR/BS was $<103.8 \mathrm{pg} / \mathrm{mL}$, and to discriminate high from nonhigh BFR/BS was $>323.0 \mathrm{pg} / \mathrm{mL}$. The best cutoff for bALP to discriminate low from nonlow BFR/BS was $<33.1 \mathrm{U} / \mathrm{L}$, and for high from nonhigh BFR/BS, $42.1 \mathrm{U} / \mathrm{L}$. Using the KDIGO practice guideline PTH values of greater than 2 but less than 9 times the upper limit of normal, sensitivity and specificity of $\mathrm{iPTH}$ level to discriminate low from nonlow turnover bone disease were $65.7 \%$ and $65.3 \%$, and to discriminate high from nonhigh were $37.0 \%$ and $85.8 \%$, respectively.

Limitations: Cross-sectional design without consideration of therapy. Potential limited generalizability with samples from 4 countries.

Conclusions: The serum biomarkers $\mathrm{PTH}$, whole PTH, and bALP were able to discriminate low from nonlow BFR/BS, whereas iPTH and bALP were able to discriminate high from nonhigh BFR/BS. Prospective studies are required to determine whether evaluating trends in biomarker concentrations could guide therapeutic decisions. Am J Kidney Dis. 67(4):559-566. (c) 2016 by the National Kidney Foundation, Inc.
}

INDEX WORDS: Sensitivity and specificity; alkaline phosphatases; bone-specific alkaline phosphatase (bALP; BSAP); bone histomorphometry; chronic kidney disease-mineral bone disorder (CKD-MBD); parathyroid hormone (PTH); procollagen type $1 \mathrm{~N}$ propeptide (P1NP); renal osteodystrophy.

\section{Editorial, p. 535}

$\mathbf{C}$ hronic kidney disease (CKD) is a significant public health problem, afflicting $\sim 11 \%$ of the American adult population ${ }^{1}$ with a similar prevalence worldwide. ${ }^{2}$ Disturbances of mineral metabolism, which occur in nearly all patients with CKD stages 3 to $5 \mathrm{D}$, are associated with bone loss and fractures, cardiovascular disease, inflammation, abnormal immune function, and increased mortality. ${ }^{3}$ Mineral abnormalities and renal osteodystrophy in CKD and more recently, their association with extraosseous calcifications, have been topics of great interest and controversy. ${ }^{4-8}$

In 2006, KDIGO (Kidney Disease: Improving Global Outcomes), an international initiative with a key mission of developing clinical practice guidelines concerning CKD, published a position statement proposing a new approach to classifying bone and mineral disorders termed CKD-mineral and bone disorder (CKD-MBD). ${ }^{9}$ This was defined as a systemic disorder, with renal osteodystrophy being redefined as one
From the ${ }^{1}$ NorthShore University HealthSystem, University of Chicago, Evanston, IL; ${ }^{2}$ University Hospital of Caracas, Caracas, Venezuela; ${ }^{3}$ University of São Paulo School of Medicine; ${ }^{4}$ Federal University of São Paulo, São Paulo, Brazil; ${ }^{5}$ University of Kentucky Medical Center, Lexington, KY; ${ }^{6}$ Hospital Curry Cabral, Faculdade de Ciências MédicasUniversidade Nova de Lisboa, Lisbon, Portugal; ${ }^{7}$ University of Antwerp, Antwerp, Belgium; ${ }^{8}$ Inserm U 1088, UFR Médecinel Pharmacie, UPJV, Amiens, France; ${ }^{9}$ National Kidney Foundation, New York, NY; and ${ }^{10}$ Indiana University School of
Medicine and Roudebush Veterans Administration Hospital, Indianapolis, IN.

Received June 6, 2014. Accepted in revised form June 15, 2015. Originally published online August 25, 2015.

Address correspondence to Stuart M. Sprague, DO, NorthShore University HealthSystem University of Chicago, 2650 Ridge Avenue, Evanston, IL 60201. E-mail: stuartmsprague@gmail.com (C) 2016 by the National Kidney Foundation, Inc. $0272-6386$

http://dx.doi.org/10.1053/j.ajkd.2015.06.023 
of the components of CKD-MBD, and the recommendation was to only use this term to delineate bone histopathologic findings. The gold standard for the diagnosis and specific classification of renal osteodystrophy is a bone biopsy with bone histomorphometry. ${ }^{9,10}$ There is heterogeneity of histologic abnormalities observed in patients with $\mathrm{CKD},{ }^{11}$ and patients may develop different lesions as CKD progresses. The classic description of the histologic abnormalities includes hyperparathyroid bone disease, adynamic bone disease, osteomalacia, and mixed uremic osteodystrophy. ${ }^{11-14}$ The KDIGO consensus conference agreed on a new classification of renal osteodystrophy that addresses the most important bone abnormalities, which include changes in bone turnover (T), mineralization (M), and volume (V). ${ }^{9}$ The TMV classification is consistent with the classically used classification system. ${ }^{11-14}$

The main focus on renal osteodystrophy has been to assess bone disease based on bone turnover, from low to high. Because bone turnover is a function in large part of the degree of hyperparathyroidism, circulating parathyroid hormone (PTH) levels have traditionally been used as a surrogate indicator of bone turnover. Intact PTH (iPTH), together with PTH ratio, has been studied for the diagnosis of bone turnover in dialysis patients, ${ }^{15,16}$ and differences between whites and blacks have been shown. ${ }^{16}$ However, the assay for determining PTH ratio is not widely available. Together with serum calcium, phosphorus, and total alkaline phosphatases or bone-specific alkaline phosphatase (bALP), PTH levels are used to guide the pharmacologic treatment of CKDMBD. However, using PTH levels from random serum samples as the primary criteria for defining and monitoring bone turnover alterations in CKD is an oversimplification of the complexity that governs this process. In addition, differences in intermethod (assay) PTH standards, variability, and reference ranges ${ }^{17-19}$ have led to confusion about the use of PTH as a bone biomarker. Additional bone biomarkers have also been evaluated for their predictive value in assessing renal osteodystrophy, but sample sizes of these studies were small and inconclusive. ${ }^{20,21}$ Information evaluating renal bone disease with the currently used PTH assays alone or in combination with other bone biomarkers and the utility of classifying renal osteodystrophy with the TMV system are limited. ${ }^{22,23}$ Thus, in order to better define the relative diagnostic value of various circulating biomarkers that are currently in clinical use alone or as a panel, KDIGO led an international consortium in a cross-sectional retrospective diagnostic test study. The goal of this study was to determine the predictive value of serum levels of PTH (determined by both iPTH and whole PTH assays), bALP, and amino-terminal propeptide of type 1 procollagen (P1NP) as markers of bone turnover.

\section{METHODS}

\section{Study Population and Data Collection}

Data and serum samples were obtained from clinical programs in Brazil, Portugal, Turkey, and Venezuela. All patients from the 4 study sites who had been treated with dialysis for at least 3 months and had a reported histomorphometric analysis of a bone biopsy specimen and stored serum drawn within 30 days of the acquisition of the bone biopsy specimen were included in the study. All blood specimens were obtained from November 1993 through June 2007 and stored frozen at below $-20^{\circ} \mathrm{C}$ until analyzed in November 2008. Additional data collected included demographics (age, sex, ethnicity, country of origin, and dialysis modality and vintage), biopsy technique, health history (cause of CKD, time since first diagnosis of CKD, history of diabetes mellitus, parathyroidectomy, or previous kidney transplantation), biochemical parameters (calcium, phosphorus, and iPTH), and treatment information (vitamin $\mathrm{D}$, type of vitamin $\mathrm{D}$, phosphate binder, and type of binder) at the time of the biopsy (Table 1).

\section{Serum Biochemistry}

All serum biomarkers were measured by a single central laboratory (Nordic Biochemical Research Laboratory, Herlev, Denmark). Reference ranges for P1NP were 13.9 to $85.5 \mathrm{ng} / \mathrm{mL}$ for men, 15.1 to $58.6 \mathrm{ng} / \mathrm{mL}$ for premenopausal women, and 20.3 to $76.3 \mathrm{ng} / \mathrm{mL}$ for postmenopausal women. The laboratory measured iPTH using a chemiluminescence immunoassay on a Roche Elecsys 2010 Analyzer; this assay detects both iPTH and a fragment containing amino acids 7 to 84 ; the reference range is 15.0 to $65.0 \mathrm{pg} / \mathrm{mL}$. The laboratory measured whole PTH with an immunoradiometric assay kit from Scantibodies Laboratories; this assay is specific for PTH isoforms containing amino acids 1 to 84 , and the reference range is 6.0 to $32.0 \mathrm{pg} / \mathrm{mL}$. bALP was measured by an immunoassay from Quidel (reference range, 15.0-41.3 U/L for men, 14.2-42.7 U/L for postmenopausal women, and 11.6-29.6 U/ $\mathrm{L}$ for premenopausal women). All serum samples were stored at below $-20^{\circ} \mathrm{C}$ for various periods. Specimen collection and storage condition characteristics, temperature settings $\left(-80^{\circ} \mathrm{C}\right.$ vs $\left.-20^{\circ} \mathrm{C}\right)$, and specimen age were recorded.

\section{Bone Biopsy and Histomorphometry}

Histologic interpretation of bone biopsy specimens were completed at the Bone Diagnostic and Research Laboratory, University of Kentucky, Lexington (for biopsies from patients from Turkey); Federal University of São Paulo, São Paulo, Brazil; University of São Paulo Renal Physiopathology Laboratory, São Paulo, Brazil (for biopsies from Brazil and Portugal); and University Hospital of Caracas, Caracas, Venezuela (for biopsies from Venezuela) The histomorphometric parameter used for analysis of bone turnover is bone formation rate/bone surface (BFR/BS). Each laboratory used their normative data to classify BFR/BS as either low, normal, or high (Table S1, available as online supplementary material).

\section{Statistical Analysis}

Descriptive statistics were presented as mean \pm standard deviation and median; frequency and percentage were presented for data with tabulation. Correlations between biomarkers were assessed by computing a Spearman correlation coefficient. Analysis of variance (ANOVA) or nonparametric counterpart KruskalWallis test was used to detect between-group difference; post hoc pairwise comparison was Bonferroni adjusted (for ANOVA) or by Terpstra permutation test (for nonparametric test). Logistic regression analysis was conducted to derive the area under the receiver operating characteristic (ROC) curve (AUROC) to determine the diagnostic ability for bone turnover of the biomarkers. AUROC $>0.7$ is very good, $>0.8$ is excellent, and $>0.9$ is 
Table 1. Patient Characteristics at Time of Bone Biopsy

\begin{tabular}{|c|c|c|c|c|c|}
\hline & Total & Portugal & Venezuela & Brazil & Turkey \\
\hline No. analyzed & 492 & 89 & 114 & 156 & 133 \\
\hline Female sex & $218(44.3)$ & $35(39)$ & $52(45.6)$ & $54(34.6)$ & $77(57.9)$ \\
\hline Age, y & $49.5 \pm 15.1$ & $48.0 \pm 14.1$ & $40.3 \pm 14.9$ & $49.5 \pm 12.9$ & $57.4 \pm 14.0$ \\
\hline Dialysis vintage, $y$ & $4.74 \pm 3.7$ & $7.27 \pm 4.9$ & $4.39 \pm 3.3$ & $3.89 \pm 2.7$ & $4.15 \pm 3.3$ \\
\hline Peritoneal dialysis & $7(1.4)$ & $1(1)$ & $6(5.3)$ & $0(0)$ & $0(0)$ \\
\hline \multicolumn{6}{|l|}{ Cause of ESRD } \\
\hline Hypertension & $112(22.8)$ & $22(25)$ & $27(23.7)$ & $45(28.8)$ & $18(13.5)$ \\
\hline Glomerulonephritis & $78(15.8)$ & $20(23)$ & $21(18.4)$ & $26(16.7)$ & $11(13.5)$ \\
\hline Diabetic nephropathy & $46(9.3)$ & $0(0)$ & $6(5.3)$ & 19 (12.2) & $21(15.8)$ \\
\hline Chronic interstitial nephritis & $33(6.7)$ & $11(12)$ & $11(9.6)$ & $9(5.8)$ & $2(1.5)$ \\
\hline Polycystic kidney disease & $28(5.7)$ & $9(10)$ & $3(2.6)$ & $7(4.5)$ & $9(6.8)$ \\
\hline Other/unknown & $193(39.2)$ & $25(28)$ & $46(40.4)$ & $50(32.1)$ & $72(54.1)$ \\
\hline Serum calcium, mg/dL & $9.51 \pm 1.03$ & $9.79 \pm 0.94$ & $8.86 \pm 1.10$ & $10.12 \pm 0.76$ & $9.12 \pm 0.81$ \\
\hline Serum phosphorus, mg/dL & $5.77 \pm 1.93$ & $6.15 \pm 1.95$ & $4.90 \pm 2.0$ & $6.63 \pm 1.78$ & $5.13 \pm 1.53$ \\
\hline Serum iPTH, pg/mL & $542 \pm 642$ & $812 \pm 698$ & $942 \pm 912$ & $390 \pm 330$ & $216 \pm 247$ \\
\hline History of diabetes & $58(11.8)$ & $1(1)$ & $12(10.5)$ & $21(13.5)$ & $24(18.0)$ \\
\hline History of kidney transplantation & $46(9.3)$ & $11(12)$ & $16(14.0)$ & $11(7.0)$ & $8(6.0)$ \\
\hline Parathyroidectomy & $5(1.0)$ & $1(1)$ & $2(1.8)$ & $0(0)$ & $2(1.5)$ \\
\hline Vitamin D therapy ${ }^{\mathrm{a}}$ & $143(29.1)$ & $40(45)$ & $35(30.7)$ & 67 (42.9) & $1(0.7)$ \\
\hline \multicolumn{6}{|l|}{ Phosphate binders } \\
\hline Calcium-based & $379(77.0)$ & $56(63)$ & $103(90.4)$ & $111(71.2)$ & $109(82.0)$ \\
\hline Aluminum salt & $52(10.6)$ & $44(49)$ & $5(4.4)$ & $3(1.9)$ & $0(0)$ \\
\hline Sevelamer $\mathrm{HCl}$ & $42(8.5)$ & $0(0)$ & $0(0)$ & $41(26.3)$ & $1(0.7)$ \\
\hline Corticosteroids & $24(4.9)$ & $3(3)$ & $21(18.4)$ & $0(0)$ & $0(0)$ \\
\hline Immunosuppressives & $20(4.1)$ & $3(3)$ & 17 (14.9) & $0(0)$ & $0(0)$ \\
\hline
\end{tabular}

Note: Unless otherwise indicated, values for categorical variables are given as number (percentage); values for continuous variables, as mean \pm standard deviation. None of the study participants were receiving cinacalcet, lanthanum carbonate phosphate binder, or hormone replacement therapy at the time of the biopsy. Only 1 patient (Venezuela) was prescribed a bisphosphonate at the time of the biopsy.

Abbreviations: ESRD, end-stage renal disease; iPTH, intact parathyroid hormone.

${ }^{a}$ Calcitriol was the only prescribed vitamin $D$ therapy in the study participants.

distinguished. ${ }^{24}$ The best cutoff on the AUROC curve was determined by Youden J statistic. The optimal cutoff is the threshold that maximizes the distance to the identity (diagonal) line. ${ }^{25} P<0.05$ is regarded as statistically significant. SAS, version 9.2 (SAS Institute Inc), and R version 3.1.1 (R Foundation for Statistical Computing) were used to carry out all statistical analyses.

\section{RESULTS}

\section{Study Participants}

Of 610 dialysis patient samples initially identified for this analysis, 492 had an adequate quantity of stored serum and biopsy data to be included in analyses (Fig 1). Basic demographic data for the 492 dialysis patients from Brazil, Portugal, Turkey, and Venezuela who underwent bone biopsy and had simultaneous serum samples obtained and stored frozen are presented in Table 1. There was a strong correlation $(r=0.927$; $P<0.001$ ) between serum PTH values determined with iPTH and whole PTH assays (Figs 2 and S1), with whole PTH levels being 40\% of iPTH values.

\section{Biomarker Assessment of Bone Turnover}

Bone turnover based on BFR/BS reference values of the participating laboratories was defined as being low, normal, or high. There was a positive correlation noted between each bone marker and BFR/BS (Table S2). Of 492 patients analyzed, there were 289 with low bone turnover (59\%), 120 with normal bone

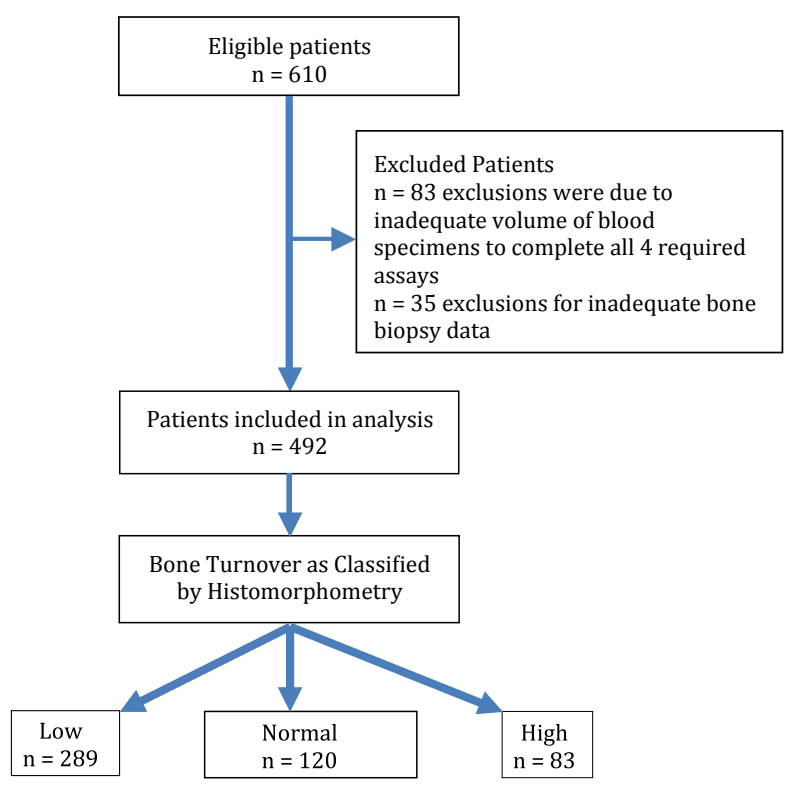

Figure 1. Flow diagram of patient samples. 


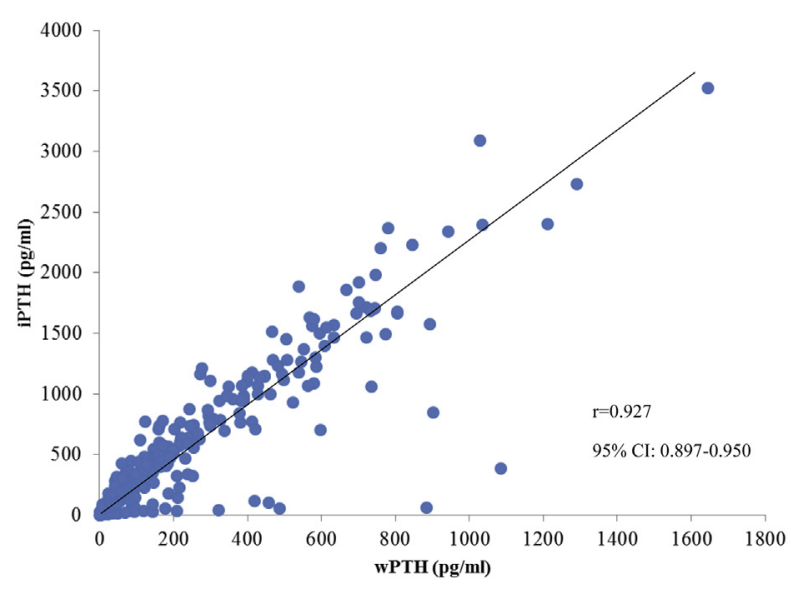

Figure 2. Correlation of parathyroid hormone (PTH) assays. The correlation between serum intact (iPTH) and serum whole PTH (wPTH) values measured by the central laboratory. There was a strong correlation $(r=0.927 ; 95 \%$ confidence interval $[\mathrm{Cl}$, 0.897-0.950; $P<0.001, \mathrm{n}=492)$ between serum $\mathrm{PTH}$ values determined with iPTH and wPTH assays.

turnover $(24 \%)$, and 83 with high bone turnover (17\%). Levels of iPTH, whole PTH, bALP, and P1NP stratified by bone turnover (low, normal, or high) are shown in Table 2.

ROC curves were developed to determine whether the bone biomarkers could discriminate between clinically important differences of low bone turnover from nonlow turnover and high bone turnover from nonhigh turnover (Table 3). To predict low turnover, these data suggest that the optimal cutoff value of iPTH of $<103.8 \mathrm{pg} / \mathrm{mL}$ would have an AUROC of 0.701, whereas the optimal cutoff of whole PTH $<48.0 \mathrm{pg} / \mathrm{mL}$ would correspond to AUROC of 0.712. bALP level $<33.1 \mathrm{U} / \mathrm{L}$ would have an AUROC of 0.757 to predict low bone turnover, whereas an optimal P1NP value $<498.9 \mathrm{ng} / \mathrm{mL}$ did not adequately predict low bone turnover (AUROC $=0.650)$. To predict high turnover, an optimal iPTH level would be $>323.0 \mathrm{pg} / \mathrm{mL}$ with an AUROC of 0.724 , whereas the whole PTH assay did not adequately predict high bone turnover $($ AUROC $=0.678)$. bALP level $>42.1 \mathrm{U} / \mathrm{L}$ would have an AUROC of 0.711 to predict high bone turnover, and a P1NP level $>621.1 \mathrm{ng} / \mathrm{mL}$ would have an AUROC of 0.743 to predict high bone turnover (Table 3).

The combination of iPTH and bALP levels generated slightly better ability to discriminate high from nonhigh bone turnover (AUROC $=0.718$ ) than whole PTH and bALP levels. Discrimination between low and nonlow bone turnover was also slightly improved with the combination of iPTH and bALP or whole PTH and bALP levels in combination (AUROCs $=0.718$ and 0.743 , respectively). Thus, although there were differences between bone turnover and iPTH, whole PTH, and bALP levels across bone turnover classifications, no biomarker singly or in combination was robust enough to diagnose low, normal, and high bone turnover.

Country, calcium use, and age were found to be significantly associated with BFR/BS discrimination of low versus nonlow bone turnover in a univariate model. However, adjusting for these parameters in multivariate analysis, both iPTH and whole PTH levels remained significantly predictive $(P<0.001)$ to discriminate low from nonlow bone turnover (Table S3). Country, diabetes as primary diagnosis, use of non-calcium-based phosphate binder, no use of phosphate binder, and dialysis vintage (years) were found to be significantly associated with BFR/BS discrimination of high versus nonhigh bone turnover in a univariate model (all $P<0.05$ ). However, after adjusting for these factors in multivariate analysis, both iPTH and whole PTH levels remained significantly predictive $(P<0.001)$ to discriminate high from nonhigh bone turnover (Table S4).

Clinical practice guidelines have recommended the use of ranges of iPTH in order to optimize therapies. Thus, in addition to evaluating the diagnostic accuracy of iPTH with or without bALP level as a continuous measure, we also assessed the diagnostic utility of using the iPTH stratification scheme based on the NKF-KDOQI (National Kidney Foundation-Kidney Disease Outcomes Quality Initiative) iPTH target level (150-300 pg/mL) and the KDIGO iPTH or whole PTH level range (2-9 times the upper limit of normal),

Table 2. Discrimination of Bone Turnover by Serum Biochemistry

\begin{tabular}{lcccr}
\hline & $\begin{array}{c}\text { Low } \\
(\mathbf{n}=\mathbf{2 8 9})\end{array}$ & $\begin{array}{c}\text { Normal } \\
(\mathbf{n = 1 2 0})\end{array}$ & $\begin{array}{c}\text { High } \\
(\mathbf{n}=\mathbf{8 3})\end{array}$ & Reference Range \\
\hline $\mathrm{iPTH}, \mathrm{pg} / \mathrm{mL}$ & $68.2[23.2-186.3]$ & $180.7[50.0-717.9]$ & $382.6[139.5-865.5]$ & $15.0-65.0$ \\
$\mathrm{WPTH}, \mathrm{pg} / \mathrm{mL}$ & $24.2[8.1-68.4]$ & $81.4[27.3-299.0]$ & $106.0[36.7-369.4]$ & $6.0-32.0$ \\
$\mathrm{bALP}, \mathrm{U} / \mathrm{L}$ & $28.2[18.0-46.2]$ & $33.7[60.0-118.0]$ & $63.3[42.3-116.8]$ & $11.6-42.7$ \\
$\mathrm{P} 1 \mathrm{NP}, \mathrm{ng} / \mathrm{mL}$ & $348.3[183.1-599.6]$ & $483.7[207.1-786.4]$ & $787.0[523.7-992.2]$ & $13.9-85.5$ \\
\hline
\end{tabular}

Note: Values are given as median [interquartile range]. Assignment to low, normal, and high bone turnover categories was based on assessment of bone formation rate/bone surface. Normal values for each laboratory are in Table S1. Terpstra permutation test $P$ values for all markers $<0.001$.

Abbreviations: bALP, bone-specific alkaline phosphatase; iPTH, intact parathyroid hormone; P1NP, amino-terminal propeptide of type 1 procollagen; wPTH, whole parathyroid hormone. 
Table 3. AUROCs of Circulating Bone Biomarkers to Distinguish High and Low Bone Turnover From Nonhigh and Nonlow Bone Turnover as Assessed by BFR/BS

\begin{tabular}{|c|c|c|c|}
\hline $\begin{array}{l}\text { Blood Sample } \\
\text { Marker }\end{array}$ & $\mathbf{N}$ & AUROC $(95 \% \mathrm{Cl})$ & $\begin{array}{l}\text { Best } \\
\text { Cutoff }\end{array}$ \\
\hline \multicolumn{4}{|c|}{ Low vs Nonlow } \\
\hline iPTH, pg/mL & 280 vs 196 & $0.701(0.653-0.750)$ & 103.8 \\
\hline wPTH, pg/mL & 260 vs 180 & $0.712(0.662-0.761)$ & 48.0 \\
\hline bALP, U/L & 273 vs 190 & $0.757(0.713-0.801)$ & 33.1 \\
\hline $\mathrm{P} 1 \mathrm{NP}, \mathrm{ng} / \mathrm{mL}$ & 280 vs 1,197 & $0.650(0.599-0.701)$ & 498.9 \\
\hline $\begin{array}{l}\text { Combined iPTH }+ \\
\text { bALP }\end{array}$ & 272 vs 188 & $0.718(0.670-0.767)$ & NA \\
\hline $\begin{array}{l}\text { Combined } \\
\text { wPTH + bALP }\end{array}$ & 257 vs 174 & $0.743(0.695-0.790)$ & NA \\
\hline \multicolumn{4}{|c|}{ High vs Nonhigh } \\
\hline iPTH, pg/mL & 81 vs 395 & $0.724(0.663-0.786)$ & 323.0 \\
\hline wPTH, pg/mL & 75 vs 365 & $0.678(0.611-0.746)$ & 61.4 \\
\hline bALP, U/L & 77 vs 386 & $0.711(0.655-0.767)$ & 42.1 \\
\hline $\mathrm{P} 1 \mathrm{NP}, \mathrm{ng} / \mathrm{mL}$ & 81 vs 396 & $0.743(0.689-0.797)$ & 621.1 \\
\hline $\begin{array}{l}\text { Combined iPTH + } \\
\text { bALP }\end{array}$ & 76 vs 384 & $0.718(0.658-0.779)$ & NA \\
\hline $\begin{array}{l}\text { Combined } \\
\text { wPTH + bALP }\end{array}$ & 72 vs 359 & $0.691(0.628-0.725)$ & NA \\
\hline
\end{tabular}

Abbreviations: AUROC, area under the receiver operating characteristic curve; bALP, bone-specific alkaline phosphatase; $\mathrm{BFR} / \mathrm{BS}$, bone formation rate/bone surface; $\mathrm{Cl}$, confidence interval; iPTH, intact parathyroid hormone; NA, not available; $\mathrm{P} 1 \mathrm{NP}$, amino-terminal propeptide of type 1 procollagen; wPTH, whole parathyroid hormone.

respectively. ${ }^{21,26}$ The sensitivity and specificity of discriminating high and low bone turnover based on these PTH values is shown in Table 4. The utility of PTH level in differentiating high bone turnover from nonhigh turnover using the iPTH cutoff for NKFKDOQI $(>300 \mathrm{pg} / \mathrm{mL})$ and KDIGO $(>9$ times the upper limit of normal) had relatively high specificity, with use of the KDIGO value being slightly more specific. However, both guideline cutoff values had low sensitivity. The positive and negative predictive values for PTH according to KDIGO and NKF-KDOQI guidelines are summarized in Table 5.

In summary, although there was significant ROCbased discrimination for iPTH plus bALP levels in differentiating low from nonlow bone turnover and high from nonhigh bone turnover, these biomarkers did not reach acceptable levels of discrimination for use in single-time-point diagnosis in an individual patient. The other biomarkers did not add diagnostic value.

\section{DISCUSSION}

This study represents what is to our knowledge one of the 2 largest bone biopsy studies ever reported, with the goal to determine the usefulness of circulating biomarker levels for predicting bone histopathology in an international setting (the other was an analysis of 630 bone biopsies $^{23}$ ). For more than 30 years, prevention and treatment of the disturbances of bone and mineral metabolism of CKD have focused
Table 4. Sensitivity and Specificity of PTH Using Cutoffs of Published Clinical Practice Guidelines to Predict Bone Turnover as Assessed by BFR/BS

\begin{tabular}{lcc}
\hline & Sensitivity & Specificity \\
\hline & & \\
NKF-KDOQI guideline cutoffs & & \\
iPTH $<150 \mathrm{pg} / \mathrm{mL}$ for low turnover & $68.6 \%$ & $61.2 \%$ \\
iPTH $>300 \mathrm{pg} / \mathrm{mL}$ for high turnover & $58.0 \%$ & $77.7 \%$ \\
KDIGO guideline cutoffs & & \\
iPTH $<2 \times$ ULN for low turnover & $65.0 \%$ & $67.3 \%$ \\
iPTH $>9 \times$ ULN for high turnover & $37.0 \%$ & $85.8 \%$ \\
KDIGO guideline cutoffs & & \\
WPTH $<2 \times$ ULN for low turnover & $73.5 \%$ & $56.7 \%$ \\
WPTH $>9 \times$ ULN for high turnover & $30.7 \%$ & $87.9 \%$ \\
\hline
\end{tabular}

Abbreviations: BFR/BS, bone formation rate/bone surface; iPTH, intact parathyroid hormone; KDIGO, Kidney Disease: Improving Global Outcomes; NKF-KDOQI, National Kidney Foundation-Kidney Disease Outcomes Quality Initiative; PTH, parathyroid hormone; wPTH, whole parathyroid hormone; ULN, upper limit of normal.

on secondary hyperparathyroidism. Current therapies to treat hyperparathyroidism are used to improve bone remodeling with the ultimate goal of reducing fracture risk. The present study demonstrates that use of PTH level to assess bone turnover continues to be the best parameter of those tested.

Although PTH and bALP levels performed better than the other parameters, our study demonstrated that both biomarkers provided only suboptimal acceptable discriminating ability for the assessment of bone turnover because the AUROC was $<0.80$. The combination of PTH and bALP levels offered minimal additional discrimination and one could argue that bALP level alone was at least as good. This is consistent with previous observations. ${ }^{27,28}$

In past studies, the discriminatory ability of PTH level to predict bone turnover has been obtained by using the Nichols iPTH assay, which is no longer available. Currently available second-generation immunometric PTH assays show variable detection of PTH fragments in addition to $\mathrm{PTTH}$, which has been shown to modify the interpretation of "active" PTH values. However, we did not find clinical differences in the AUROC using iPTH (secondgeneration) compared to whole PTH (third-generation) assays. These results are not surprising if one considers that bone metabolism in CKD is affected by an intricate pathophysiologic process involving several humoral and local factors besides PTH.

Current strategies have focused on the use of PTH as a surrogate for bone turnover, and treatment is mostly aimed at lowering PTH levels with vitamin D receptor activators ${ }^{29}$ or calcimimetics. ${ }^{30}$ Because of concerns about the oversuppression of bone remodeling, the recent KDIGO clinical practice guideline has broadened the range of target or goal PTH levels, in 
Table 5. Utility of NKF-KDOQI and KDIGO iPTH Thresholds for Diagnostic Decision Making

\begin{tabular}{|c|c|c|c|c|c|c|c|c|}
\hline & \multicolumn{4}{|c|}{ NKF-KDOQI ${ }^{\mathrm{a}}$} & \multicolumn{4}{|c|}{ KDIGO $^{\mathrm{b}}$} \\
\hline & Sensitivity & Specificity & PPV & NPV & Sensitivity & Specificity & PPV & NPV \\
\hline $\begin{array}{l}\text { Differentiating low from nonlow } \\
\text { turnover bone disease, or "When } \\
\text { do I stop therapy?" }\end{array}$ & $68.5 \%$ & $61.2 \%$ & $71.6 \%$ & $57.7 \%$ & $65.7 \%$ & $65.3 \%$ & $73 \%$ & $57 \%$ \\
\hline $\begin{array}{l}\text { Differentiating high from nonhigh } \\
\text { turnover bone disease, or "When } \\
\text { do I start therapy?" }\end{array}$ & $58.0 \%$ & $77.7 \%$ & $34.8 \%$ & $90 \%$ & $37.0 \%$ & $85.8 \%$ & $34.9 \%$ & $86.9 \%$ \\
\hline $\begin{array}{l}\text { Abbreviations: iPTH, intact parat } \\
\text { Kidney Foundation-Kidney Disease } \\
\text { a Using serum iPTH }<150 \mathrm{pg} / \mathrm{mL} \\
\text { bUsing serum }\end{array}$ & $\begin{array}{l}\text { id hormon } \\
\text { comes Qu } \\
\text { ower and }\end{array}$ & $\begin{array}{l}\text { KDIGO, } \\
\text { ty Initiativ } \\
00 \mathrm{pg} / \mathrm{mL}\end{array}$ & $\begin{array}{l}\text { Dis } \\
\text { DV, ne } \\
\text { upper }\end{array}$ & $\begin{array}{l}\text { Impr } \\
\text { le pred } \\
\text { hold. }\end{array}$ & $\begin{array}{l}\text { g Global } \\
\text { e value; }\end{array}$ & $\begin{array}{l}\text { comes; } \\
\text { positive }\end{array}$ & $\begin{array}{l}\text {-KDOQ } \\
\text { dictive }\end{array}$ & $\begin{array}{l}\text { Vation } \\
\text { lue. }\end{array}$ \\
\hline
\end{tabular}

part to reduce the risk of treating patients with low turnover bone disease or to induce it, as shown by bone histomorphometry, despite relatively high serum PTH levels. ${ }^{21}$ Therefore, diagnostic accuracy of PTH as a surrogate for bone turnover is of paramount importance, although this study did not test the recommended use of trends to monitor therapy. In the present study, bone biopsies with simultaneously obtained and stored serum samples were analyzed to determine the predictive value of PTH level for assessment of renal osteodystrophy by bone histomorphometry. Due to controversies regarding PTH assays and the variable results obtained from different assays, ${ }^{19}$ biopsies were analyzed based on stored serum collected at the time of the biopsy that was reanalyzed for both iPTH, measured by chemiluminescence immunoassay (Roche Elecsys 2010 Analyzer), and whole PTH (ie, amino acids 1-84), measured by immunoradiometric assay (Scantibodies Laboratories). The pooling of specimens across the globe allowed for adequate power to test PTH and combinations of bone biomarkers. Based on an AUROC $\geq 0.70$, serum iPTH values situated in either the NKF-KDOQI iPTH target ranges $(150-300 \mathrm{pg} / \mathrm{mL})$ or the KDIGO PTH target ranges $(>2$ or $<9$ times the upper limit of normal) had relatively high specificity, but low sensitivity to differentiate high turnover from nonhigh turnover as assessed by bone histomorphometric analysis. Measuring serum whole PTH did not improve specificity or sensitivity. Unfortunately, the addition of bALP or other biomarkers did not prove to be clinically useful beyond the diagnostic value of PTH in the assessment of bone turnover. Consistent with other studies, serum PTH levels correlated with bone turnover ${ }^{14,23,31-33}$; however, to date, PTH levels have not been found to be a strong discriminator among low, normal, and high bone turnover. The present analysis demonstrates that iPTH can be used to discriminate low turnover from nonlow turnover with iPTH values consistent with those recommended by both NKFKDOQI and KDIGO (Table 4). ${ }^{21,26}$
The other clinical dilemma is when to stop PTHlowering therapies. Clearly understanding when low turnover bone disease is present without having to rely on a bone biopsy would be very useful to reliably guide therapy in clinical practice. The present study found that measurement of whole PTH, but not iPTH, using either clinical practice guideline cutoffs had relatively high sensitivity, but not specificity for discriminating low turnover from nonlow turnover disease on bone biopsy. Using the iPTH assay and KDIGO clinical practice guideline (Table 5), the negative predictive value for discriminating low from nonlow turnover to answer the question of when to stop therapy was $57 \%$, with a positive predictive value of $73 \%$. To answer the question "When do I start therapy to treat high turnover bone disease?" using the iPTH assay, the negative predictive value for discriminating high from nonhigh turnover was $87 \%$, with a positive predictive value of $35 \%$. Thus, the KDIGO range is reasonable, but far from ideal, to help guide clinical practice.

The majority of individuals had low bone turnover. Malluche et $\mathrm{al}^{23}$ evaluated 630 bone biopsies and reported a similarly high percentage of patients with low bone turnover ( $58 \%$ compared to $59 \%$ in the present study) and low percentage with high turnover ( $24 \%$ compared to $17 \%$ in present study). The substantial difference in the prevalence of high turnover bone disease between studies may be due at least in part to ethnicity. In the analysis by Malluche et al, black participants were found to have a significantly higher prevalence of high turnover compared with white participants, and they had a much larger proportion of black patients than the present analysis (14\% vs 6\%). Another potential difference is in the choice of therapies, which differs by country.

In addition to the commonly used bone biomarker PTH, we assessed the ability of bALP and P1NP levels to provide additional diagnostic accuracy. Unfortunately, the inclusion of bALP level provided only limited clinically nonsignificant added value to 
that of PTH, and P1NP did not improve the diagnostic accuracy. Serum samples were of limited quantity and thus assessments of the diagnostic performance of fibroblast growth factor 23, 25-hydroxyvitamin $\mathrm{D}$, sclerostin, tartrate-resistant acid phosphatase $5 \mathrm{~b}$ (TRAP-5b), and other newer biomarkers could not be made.

A major limitation of the present study is its crosssectional design, which did not allow interpretation of the therapeutic interventions that mitigate the relationship of bone turnover with PTH and other biomarkers. Other limitations are the origin of samples from only 4 countries (with potentially limited generalizability), no data to address differences between ethnic and racial groups, high use of calciumbased phosphate binders and low use of vitamin D sterols, and low prevalence of patients with diabetes. In addition, uncertain stability of frozen blood samples may have affected results. Further, with the exception of the biopsies from Turkey, the other participants were referred for biopsy to assist in clinical management and therefore may not be generalizable to all patients with end-stage kidney disease. Finally, there was no analysis of parameters of cortical bone, which appears to be preferentially lost in patients with CKD and hyperparathyroidism. ${ }^{34}$

In conclusion, PTH measured by iPTH assay offers a reasonable assessment of bone turnover and the ability to discriminate high from nonhigh and low from nonlow turnover based on either the NKFKDOQI or KDIGO targets for PTH. This was only marginally improved with the addition of bALP and was not affected by other biomarkers. Although the AUROC $<0.7$ is suboptimal, this is not a surprising finding given the short half-life of PTH and the long duration of a bone remodeling cycle. Because these analyses could not determine whether evaluating trends in PTH levels over time could guide therapeutic decisions, further prospective studies are required. In the absence of performing a bone biopsy, iPTH measurement probably remains the best clinically available tool to discriminate the extremes of bone turnover.

The purpose of this study was to evaluate the diagnostic value of biomarkers to predict bone turnover and not to test the validity of guidelines. The guidelines were based on limited evidence, which did not include bone biopsy studies. However, the present data are consistent with recommendations proposed by KDIGO in using PTH level for the clinical assessment of bone turnover, especially at the extremes of PTH values. Trending changes in PTH levels, an issue that has not been assessed in this or other studies, may improve diagnostic precision and provide better clinical guidance for the treatment of secondary hyperparathyroidism, as suggested by KDIGO. In this context, it must also be emphasized that PTH exerts actions on many tissues other than bone and that this study does not negate the usefulness of monitoring PTH levels in dialysis patients. ${ }^{35}$

\section{ACKNOWLEDGEMENTS}

The authors thank Drs Ercan Ok, Gulay Asci, and Fatih Kircelli (Ege University School of Medicine, Turkey) for assistance with bone biopsy and data collection.

Support: This study was supported by unrestricted grants to the NKF from Abbott, Amgen, Genzyme, and Shire and was facilitated by KDIGO and the NKF, the former managing agent for KDIGO. The funding organizations had no role in the design, conduct, analysis, or interpretation of the study; writing the report; or the decision to submit it for publication.

Financial Disclosure: Dr Sprague has received funding from Abbott-AbbVie, Amgen, Cytochroma, Fresenius Medical Care, and Vifor and consulting fees from Amgen, Cytochroma, and Vifor. Dr Bellorin-Font has received honoraria from Abbott, Amgen, and Sanofi/Genzyme. Dr Jorgetti has received consulting and speaker fees from Abbott, Amgen, and Sanofi/Genzyme. Dr Carvalho has received consulting and speaker fees from AbbottAbbvie, Amgen, and Sanofi/Genzyme. Dr Malluche has received research support from Novartis, Celgene, and Vifor. Dr Ferreira has received consulting fees from Abbott-Abbvie, Amgen, Fresenius Medical Care, and Sanofi/Genzyme and speaker fees from Abbott-Abbvie, Amgen, Fresenius Medical Care, Sanofi/Genzyme, and Shire. Dr D'Haese has received funding from Amgen, Baxter, Diasorin, Fresenius Medical Care, Novartis, Shire, and Vifor. Dr Drüeke has received consulting fees from AbbottAbbVie, Amgen, Baxter, Chugai, Fresenius Medical Care, Sanofi/Genzyme, and Vifor; speaker fees from Abbott-AbbVie, Amgen, Chugai, Kirin, Sanofi/Genzyme, and Vifor; and funding from Amgen, Baxter, and Shire. The other authors declare that they have no other relevant financial interests.

Contributions: Research idea and study design: SMS, EB-F, VJ, ABC, HHM, AF, PCD'H, TBD, HD, TM, SMM; data acquisition: EB-F, VJ, ABC, HHM, AF, TM, ER; data analysis/interpretation: SMS, EB-F, VJ, ABC, HHM, AF, PCD'H, TBD, HD, SMM; statistical analysis: SMS, HD; supervision or mentorship: SMS, EB-F, TBD, SMM. Each author contributed important intellectual content during manuscript drafting or revision and accepts accountability for the overall work by ensuring that questions pertaining to the accuracy or integrity of any portion of the work are appropriately investigated and resolved. SMS takes responsibility that this study has been reported honestly, accurately, and transparently; that no important aspects of the study have been omitted; and that any discrepancies from the study as planned have been explained.

\section{SUPPLEMENTARY MATERIAL}

Table S1: Normal values of bone turnover in various laboratories.

Table S2: Spearman correlations between BFR/BS and blood biomarkers.

Table S3: Prediction for BFR/BS low vs nonlow with adjustments.

Table S4: Prediction for BFR/BS high vs nonhigh with adjustments.

Figure S1: Correlation of PTH assays using Bland-Altman analysis.

Note: The supplementary material accompanying this article (http://dx.doi.org/10.1053/j.ajkd.2015.06.023) is available at www.ajkd.org 


\section{REFERENCES}

1. Coresh J, Selvin E, Stevens LA, et al. Prevalence of chronic kidney disease in the United States. JAMA. 2007;298(17):2038-2047.

2. Zhang QL, Rothenbacher D. Prevalence of chronic kidney disease in population-based studies: systematic review. BMC Public Health. 2008;8:117.

3. Tentori F, Blayney MJ, Albert JM, et al. Mortality risk for dialysis patients with different levels of serum calcium, phosphorus, and PTH: the Dialysis Outcomes and Practice Patterns Study (DOPPS). Am J Kidney Dis. 2008;52(3):519-530.

4. Adragao T, Herberth J, Monier-Faugere MC, et al. Low bone volume - a risk factor for coronary calcifications in hemodialysis patients. Clin J Am Soc Nephrol. 2009;4(2):450-455.

5. Asci G, Ok E, Savas R, et al. The link between bone and coronary calcifications in CKD-5 patients on haemodialysis. Nephrol Dial Transplant. 2011;26(3):1010-1015.

6. Barreto DV, Barreto Fde C, Carvalho AB, et al. Association of changes in bone remodeling and coronary calcification in hemodialysis patients: a prospective study. Am J Kidney Dis. 2008;52(6): 1139-1150.

7. Filgueira A, Carvalho AB, Tomiyama $\mathrm{C}$, et al. Is coronary artery calcification associated with vertebral bone density in nondialyzed chronic kidney disease patients? Clin J Am Soc Nephrol. 2011;6(6):1456-1462.

8. Tomiyama C, Carvalho AB, Higa A, Jorgetti V, Draibe SA, Canziani ME. Coronary calcification is associated with lower bone formation rate in CKD patients not yet in dialysis treatment. J Bone Miner Res. 2010;25(3):499-504.

9. Moe S, Drueke T, Cunningham J, et al. Definition, evaluation, and classification of renal osteodystrophy: a position statement from Kidney Disease: Improving Global Outcomes (KDIGO). Kidney Int. 2006;69(11):1945-1953.

10. Malluche HH, Monier-Faugere MC. Renal osteodystrophy: what's in a name? Presentation of a clinically useful new model to interpret bone histologic findings. Clin Nephrol. 2006;65(4): 235-242.

11. Malluche HH, Faugere MC. Atlas of Mineralized Bone Histology. Basel, Switzerland: Karger AG; 1986.

12. Hruska KA, Teitelbaum SL. Renal osteodystrophy. N Engl J Med. 1995;333(3):166-174.

13. Lehmann G, Ott U, Kaemmerer D, Schuetze J, Wolf G. Bone histomorphometry and biochemical markers of bone turnover in patients with chronic kidney disease stages 3-5. Clin Nephrol. 2008;70(4):296-305.

14. Sherrard DJ, Hercz G, Pei Y, et al. The spectrum of bone disease in end-stage renal failure-an evolving disorder. Kidney Int. 1993;43(2):436-442.

15. Monier-Faugere MC, Geng Z, Mawad H, et al. Improved assessment of bone turnover by the PTH-(1-84)/large C-PTH fragments ratio in ESRD patients. Kidney Int. 2001;60(4):1460-1468.

16. Herberth J, Branscum AJ, Mawad H, Cantor T, MonierFaugere MC, Malluche HH. Intact PTH combined with the PTH ratio for diagnosis of bone turnover in dialysis patients: a diagnostic test study. Am J Kidney Dis. 2010;55(5):897-906.

17. Gardham C, Stevens PE, Delaney MP, LeRoux M, Coleman A, Lamb EJ. Variability of parathyroid hormone and other markers of bone mineral metabolism in patients receiving hemodialysis. Clin J Am Soc Nephrol. 2010;5(7):1261-1267.
18. Herberth J, Monier-Faugere MC, Mawad HW, et al. The five most commonly used intact parathyroid hormone assays are useful for screening but not for diagnosing bone turnover abnormalities in CKD-5 patients. Clin Nephrol. 2009;72(1):5-14.

19. Souberbielle JC, Boutten A, Carlier MC, et al. Inter-method variability in PTH measurement: implication for the care of CKD patients. Kidney Int. 2006;70(2):345-350.

20. Moorthi RN, Moe SM. Recent advances in the noninvasive diagnosis of renal osteodystrophy. Kidney Int. 2013;84:886-894.

21. KDIGO CKD-MBD Work Group. KDIGO clinical practice guideline for the diagnosis, evaluation, prevention, and treatment of chronic kidney disease-mineral and bone disorder (CKDMBD). Kidney Int Suppl. 2009;113:S1-S130.

22. Bakkaloglu SA, Wesseling-Perry K, Pereira RC, et al. Value of the new bone classification system in pediatric renal osteodystrophy. Clin J Am Soc Nephrol. 2010;5(10):1860-1866.

23. Malluche HH, Mawad HW, Monier-Faugere MC. Renal osteodystrophy in the first decade of the new millennium: analysis of 630 bone biopsies in black and white patients. $J$ Bone Miner Res. 2011;26(6):1368-1376.

24. Hosmer DW, Lemeshow S. Assessing the fit of the model. In: Hosmer DW, Lemeshow S, eds. Applied Logistic Regression. New York, NY: John Wiley \& Sons; 2000:143-202.

25. Youden WJ. Index for rating diagnostic tests. Cancer. 1950;3(1):32-35.

26. National Kidney Foundation. K/DOQI clinical practice guidelines for bone metabolism and disease in chronic kidney disease. Am J Kidney Dis. 2003;42(suppl 3):S1-S201.

27. Bervoets AR, Spasovski GB, Behets GJ, et al. Useful biochemical markers for diagnosing renal osteodystrophy in predialysis end-stage renal failure patients. Am J Kidney Dis. 2003;41(5): 997-1007.

28. Couttenye MM, D'Haese PC, Van Hoof VO, et al. Low serum levels of alkaline phosphatase of bone origin: a good marker of adynamic bone disease in haemodialysis patients. Nephrol Dial Transplant. 1996;11(6):1065-1072.

29. Gal-Moscovici A, Sprague SM. Use of vitamin D in chronic kidney disease patients. Kidney Int. 2010;78(2):146-151.

30. Moe SM, Cunningham J, Bommer J, et al. Long-term treatment of secondary hyperparathyroidism with the calcimimetic cinacalcet HCl. Nephrol Dial Transplant. 2005;20(10):2186-2193.

31. Qi Q, Monier-Faugere MC, Geng Z, Malluche HH. Predictive value of serum parathyroid hormone levels for bone turnover in patients on chronic maintenance dialysis. Am J Kidney Dis. 1995;26(4):622-631.

32. Salusky IB, Ramirez JA, Oppenheim W, Gales B, Segre GV, Goodman WG. Biochemical markers of renal osteodystrophy in pediatric patients undergoing CAPD/CCPD. Kidney Int. 1994;45(1):253-258.

33. Wesseling-Perry K, Pereira RC, Wang H, et al. Relationship between plasma fibroblast growth factor- 23 concentration and bone mineralization in children with renal failure on peritoneal dialysis. J Clin Endocrinol Metab. 2009;94(2):511-517.

34. Nickolas TL, Stein EM, Dworakowski E, et al. Rapid cortical bone loss in patients with chronic kidney disease. J Bone Miner Res. 2013;28(8):1811-1820.

35. Sprague SM, Moe SM. The case for routine parathyroid hormone monitoring. Clin J Am Soc Nephrol. 2013;8(2):313-318. 